\title{
Side-to-side asymmetry in absolute and relative muscle thickness of the lateral abdominal wall in cricket pace bowlers
}

\author{
B Olivier, ${ }^{1} \mathrm{PT}, \mathrm{MSc}$; V Stewart, ${ }^{1} \mathrm{PT}, \mathrm{PhD} ; \mathrm{W}$ Mckinon, ${ }^{2} \mathrm{PhD}$ \\ ${ }^{1}$ School of Therapeutic Sciences, Department of Physiotherapy, Faculty of Health Sciences, University of the Witwatersrand, Johannesburg, South Africa \\ ${ }^{2}$ School of Physiology, Faculty of Health Sciences, University of the Witwatersrand, Johannesburg, South Africa
}

Corresponding author: B Olivier (benita.olivier@wits.ac.za)

Background. The abdominal musculature plays a protective role against lower-back injury. Knowledge of the asymmetry in abdominal wall thickness in healthy, injury-free cricket pace bowlers may provide a useful platform against which pathology could be assessed and the effects of training could be evaluated.

Objective. To compare side-to-side differences in absolute muscle thickness and activity of the abdominal musculature and to compare these measurements at the start, with those at the end of a cricket season among a group of amateur pace bowlers.

Methods. This was a controlled longitudinal prospective study. Rehabilitative ultrasound imaging was used to assess abdominal muscle thickness in 26 right-handed, injury-free cricket pace bowlers at the start and at the end of a cricket season. Thickness measurements were done at rest, during an abdominal drawing-in manoeuvre (ADIM) and the active straight-leg raise (ASLR) on the left (-L) and right (-R). Results. The absolute thickness of the non-dominant obliquus abdominis internus (OI) was higher than that of the dominant OI at the start ( $p=0.001 ; \mathrm{ES}=0.87$ ) as well as at the end of the cricket season ( $p=0.001$; ES 1.09). At the start of the season, the percentage change during the ADIM, thus muscle activity, was higher for the non-dominant OI than for the dominant $\mathrm{OI}(p=0.02$; $\mathrm{ES}=0.51$ ). Absolute thickness of the dominant obliquus abdominis externus $(\mathrm{OE})$ at rest was significantly higher at the end of the season compared with the start of the season ( $p=0.0001 ; \mathrm{ES}=0.85$ ). During ASLR-R, the activity of the left transversus abdominis (TA) was significantly higher than that of the right TA during ASLR-L $(p=0.03$ ) when measured at the end of the season.

Conclusion. This study highlights the possible muscle adaptations in absolute muscle thickness and activity as a consequence of the asymmetrical bowling action.

S Afr J SM 2013;25(3):81-86. DOI:10.7196/SAJSM.377

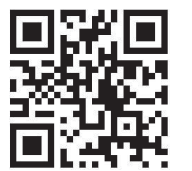

Lower-back injury is one of the most common types of injury sustained by cricket pace bowlers. ${ }^{[1]}$ The abdominal musculature plays a protective role against such injury by increasing the stability of the lumbar segmental vertebrae. ${ }^{[2]}$ The assessment of thickness of the abdominal muscles including transversus abdominis (TA), obliquus abdominis internus (OI) and oblique abdominis externus $(\mathrm{OE})$ is known to be a valid measure of size of the abdominal muscles, as well as a sensitive measure of change. Ferreira $e t$ al. ${ }^{[3]}$ found that TA and OI percentage change in thickness are valid measures of activity compared with electromyography (EMG). Rehabilitative ultrasound imaging (RUSI) is often used as a surrogate measure for activation of the abdominal muscles. Muscle thickness, as measured by rehabilitative ultrasound imaging (RUSI), is well correlated with muscle thickness measurements derived from magnetic resonance imaging (MRI) ${ }^{[4]}$

Mannion et al. ${ }^{[5]}$ and Springer et al. ${ }^{[6]}$ conducted abdominal muscle activation studies in non-sporting populations and showed that there were no differences in activation between body sides. Rankin et al. ${ }^{[7]}$ also found no statistically significant differences in muscle thickness between the left and right $\mathrm{OI}$ and $\mathrm{OE}$ when measured at rest, but a difference in TA was found where the left TA was thicker than the right in moderately active individuals. They did not specify whether the recreational activities were mainly symmetrical or asymmetrical. In contrast to the findings of the aforementioned authors, ${ }^{[5-7]}$ Hides et al.$^{[8]}$ found that the OI on the non-dominant side of elite cricketers was thicker than on the dominant side. Hides et al..$^{[8]}$ assessed a small sample size of elite cricketers, including batsmen and bowlers. It was suggested that the asymmetrical demands of the repetitive bowling action may cause some muscles to be activated preferentially and hypertrophied above other muscles. ${ }^{[8]}$

Knowledge of the asymmetry in abdominal wall thickness in healthy and injury-free cricket pace bowlers may provide a useful platform against which aetiology of injury could be assessed and training could be gauged, and could provide a benchmark to direct RUSI biofeedback interventions in the future.

The aim of this study was to compare side-to-side differences in absolute muscle thickness and muscle activation of the abdominal musculature, and to compare absolute muscle thickness and muscle activation measured at the start v. the end of a cricket season in a group of amateur fast, fast-medium and medium pace bowlers.

\section{Methods}

\section{Ethics}

Ethical clearance was granted by the Human Research Ethics Committee of the University of the Witwatersrand (reference M10430). All 
participants signed informed consent and had the right to withdraw from the study without suffering any repercussions.

\section{Participants}

Twenty-six right-handed, premier league (amateur) cricket pace bowlers were invited to participate in the study on condition that they were free of injury. Participants were tested at the start and again at the end of an 8-month cricket season.

\section{Validity and reliability}

The criterion-related validity of RUSI to measure muscle thickness has been established against MRI. ${ }^{[4]}$ A strong linear relationship is also known to exist between abdominal muscle thickness change (TA and OI) and EMG amplitude during low levels of muscle contraction (up to $30 \%$ of maximum voluntary contraction). No consistent relationship was found between $\mathrm{OE}$ thickness change and muscle contraction; therefore, this should not be used to detect muscle activity. ${ }^{[3]}$ Intrarater reliability was established by Springer et al. ${ }^{[6]}$ as being between 0.93 (95\% CI 0.86 - 0.96) and 0.99 (95\% CI 0.98 - 1.00), as well as by Rankin et al. ${ }^{[7]}$ as being 0.98 - 0.99 (95\% CI 0.91 - 1.00).

\section{Procedures}

Abdominal muscle thickness was measured using a DP-6600 digital ultrasonic imaging system (Shenzhen Mindray Bio-medical Electronics, China) with a $5 \mathrm{MHz}$ curvilinear transducer with a large footprint $(\geq 60 \mathrm{~mm})$. The first author underwent training in the use of the equipment and performed all measurements. RUSI was done in brightness (B) mode. The ultrasound echo was recorded as a crosssectional gray-scale image. ${ }^{[9]}$
Each participant was positioned in supine with their legs straight. The transducer was placed along the lateral abdominal wall along the mid-axillary line, midway between the inferior angle of the rib cage (lower border of the 11th costal cartilage) and the iliac crest in the transverse plane. ${ }^{[9]}$ The medial edge of the TA muscle was positioned at the medial edge of the ultrasound image. ${ }^{[9]}$ This is the most appropriate position as all three muscles are well represented and relatively flat and easy to measure on the image. ${ }^{[7]}$ All images were taken on the left and the right with the participant at rest at the end of relaxed expiration with the glottis open to avoid bracing. ${ }^{[9]}$ The participants were then instructed to perform the abdominal drawing-in manoeuvre (ADIM) by exhaling and gently drawing their lower abdomen towards the spine, ${ }^{[10]}$ using $20 \%$ of the maximal voluntary contraction. The following standardised instructions were given to each participant: 'take a relaxed breath in and out, hold the breath out and then draw in your lower abdomen without moving your spine.' Participants had the opportunity to practice the ADIM five times before measurements were taken. After performing the ADIM, the participants were instructed to relax their abdominal muscles completely. During the active straight-leg raise (ASLR), the bowler had to lift his leg $5 \mathrm{~cm}$ from the plinth. ${ }^{[9,11]}$ ASLR was performed on the left (-L) and right (-R). The transducer position was kept constant during all of the above. ${ }^{[9]}$ The research assistant verified proper execution of the ADIM and ASLR and recorded the frame number of each image. The thickness of TA, OI and OE was measured (in $\mathrm{mm}$ ) on each of the following images: at rest, ADIM, ASLR-L and ASLR-R. The distance between the superior and inferior hyperechoic muscle fascias of the TA, OI and OE was measured in the centre of the muscle belly by using a vertical straight

Table 1. Absolute thickness at rest and percentage change ${ }^{\star}$ in thickness in ADIM, ASLR-L and ASLR-R activity positions of the dominant v. non-dominant TA, $\mathrm{OI}$ and $\mathrm{OE}^{\dagger}$

\begin{tabular}{|c|c|c|c|c|c|c|c|c|}
\hline & \multicolumn{4}{|c|}{ Pre-season $(n=26)$} & \multicolumn{4}{|c|}{ Post-season $(n=26)$} \\
\hline & $\begin{array}{l}\text { Non- } \\
\text { dominant } \\
\text { mean }( \pm S D)\end{array}$ & $\begin{array}{l}\text { Dominant } \\
\text { mean }( \pm S D)\end{array}$ & $p$-value & $\begin{array}{l}\text { Effect size } \\
\text { Cohen's } d\end{array}$ & $\begin{array}{l}\text { Non- } \\
\text { dominant } \\
\text { mean }( \pm S D)\end{array}$ & $\begin{array}{l}\text { Dominant } \\
\text { mean }( \pm S D)\end{array}$ & $p$-value & $\begin{array}{l}\text { Effect size } \\
\text { Cohen's } d\end{array}$ \\
\hline \multicolumn{9}{|l|}{ TA } \\
\hline Rest (mm) & $4.6( \pm 1.4)$ & $4.9( \pm 1.4)$ & 0.25 & 0.17 & $4.9( \pm 1.4)$ & $5.3( \pm 1.4)$ & 0.10 & 0.35 \\
\hline ADIM \% change & $56.5( \pm 47.7)$ & $41.9( \pm 31.2)$ & 0.09 & 0.37 & $41.6( \pm 34.8)$ & $27.0( \pm 27.1)$ & 0.19 & 0.48 \\
\hline ASLR-L \% change & $17.4( \pm 34.2)$ & $14.1( \pm 30.8)$ & 0.66 & 0.10 & $15.8( \pm 25.6)$ & $5.7( \pm 21.9)$ & 0.16 & 0.44 \\
\hline ASLR-R \% change & $25.4( \pm 35.5)$ & $16.5( \pm 26.3)$ & 0.22 & 0.29 & $20.2( \pm 35.2)$ & $5.5( \pm 20.8)$ & 0.05 & 0.52 \\
\hline \multicolumn{9}{|l|}{ OI } \\
\hline Rest (mm) & $14.2( \pm 4.1)$ & $11.4( \pm 2.4)$ & $0.00^{\dagger}$ & 0.87 & $15.2( \pm 3.4)$ & $11.9( \pm 2.7)$ & $0.00^{\dagger}$ & 1.09 \\
\hline ADIM \% change & $13.0( \pm 20.2)$ & $2.7( \pm 21.6)$ & $0.02^{\dagger}$ & 0.51 & $11.1( \pm 15.5)$ & $7.6( \pm 13.5)$ & 0.37 & 0.24 \\
\hline ASLR-L \% change & $9.8( \pm 27.7)$ & $1.5( \pm 20.1)$ & 0.19 & 0.35 & $14.1( \pm 19.3)$ & $7.7( \pm 21.5)$ & 0.14 & 0.32 \\
\hline ASLR-R \% change & $8.6( \pm 21.4)$ & $5.7( \pm 20.6)$ & 0.58 & 0.14 & $4.0( \pm 24.0)$ & $8.9( \pm 19.8)$ & 0.40 & 0.23 \\
\hline \multicolumn{9}{|l|}{$\mathrm{OE}$} \\
\hline Rest (mm) & $6.4( \pm 2.3)$ & $6.0( \pm 1.4)$ & 0.33 & 0.25 & $7.4( \pm 2.6)$ & $7.5( \pm 2.1)$ & 0.76 & 0.05 \\
\hline \multicolumn{9}{|c|}{$\begin{array}{l}\mathrm{TA}=\text { transversus abdominis; } \mathrm{OI}=\text { oblique abdominis internus; } \mathrm{OE}=\text { oblique abdominis externus; } \mathrm{ADIM}=\text { abdominal drawing-in manoeuvre; ASLR-L = active straight-leg raise (left); } \\
\text { ASLR- } \mathrm{R}=\text { active straight-leg raise (right); } \mathrm{SD}=\text { standard deviation. }\end{array}$} \\
\hline
\end{tabular}


Table 2. Absolute thickness at rest and percentage change* in thickness in ADIM, ASLR-L and ASLR-R activity positions of TA, OI and $\mathrm{OE}$ at the start $\mathrm{v}$. the end of a cricket season $(N=24)^{\dagger}$

\begin{tabular}{|c|c|c|c|c|c|c|c|c|}
\hline & \multicolumn{4}{|c|}{ Non-dominant } & \multicolumn{4}{|c|}{ Dominant } \\
\hline & $\begin{array}{l}\text { Pre-season } \\
\text { mean }( \pm S D)\end{array}$ & $\begin{array}{l}\text { Post-season } \\
\text { mean }( \pm \mathrm{SD})\end{array}$ & $p$-value & $\begin{array}{l}\text { Effect size } \\
\text { Cohen's } d\end{array}$ & $\begin{array}{l}\text { Pre-season } \\
\text { mean }( \pm \mathrm{SD})\end{array}$ & $\begin{array}{l}\text { Post-season } \\
\text { mean }( \pm S D)\end{array}$ & $p$-value & $\begin{array}{l}\text { Effect size } \\
\text { Cohen's } d\end{array}$ \\
\hline \multicolumn{9}{|l|}{ TA } \\
\hline Rest (mm) & $4.6( \pm 1.1)$ & $4.9( \pm 1.4)$ & 0.13 & 0.22 & $4.9( \pm 1.3)$ & $5.3( \pm 1.4)$ & 0.07 & 0.38 \\
\hline ADIM \% change & $55.8( \pm 46.2)$ & $41.6( \pm 34.8)$ & 0.13 & 0.35 & $41.7( \pm 32.3)$ & $27.0( \pm 27.1)$ & 0.07 & 0.51 \\
\hline ASLR-L \% change & $17.0( \pm 35.4)$ & $15.8( \pm 26.6)$ & 0.88 & 0.04 & $13.1( \pm 31.7)$ & $5.7( \pm 21.9)$ & 0.35 & 0.28 \\
\hline ASLR-R \% change & $23.8( \pm 34.8)$ & $20.2( \pm 35.2)$ & 0.67 & 0.11 & $16.7( \pm 27.3)$ & $5.5( \pm 20.8)$ & 0.06 & 0.47 \\
\hline \multicolumn{9}{|l|}{ OI } \\
\hline Rest (mm) & $14.1( \pm 3.9)$ & $15.2( \pm 3.4)$ & 0.12 & 0.32 & $11.3( \pm 2.3)$ & $11.9( \pm 2.7)$ & 0.28 & 0.24 \\
\hline ADIM \% change & $12.6( \pm 20.4)$ & $11.1( \pm 15.5)$ & 0.75 & 0.08 & $2.0( \pm 22.1)$ & $7.6( \pm 13.5)$ & 0.25 & 0.31 \\
\hline ASLR-L \% change & $9.7( \pm 28.34)$ & $14.1( \pm 19.3)$ & 0.48 & 0.19 & $0.03( \pm 20.2)$ & $7.7( \pm 21.5)$ & 0.07 & 0.38 \\
\hline ASLR-R \% change & $8.7( \pm 20.7)$ & $4.0( \pm 24.0)$ & 0.45 & 0.21 & $4.7( \pm 18.6)$ & $8.9( \pm 19.8)$ & 0.41 & 0.22 \\
\hline \multicolumn{9}{|l|}{$\mathrm{OE}$} \\
\hline Rest (mm) & $6.5( \pm 2.3)$ & $7.4( \pm 2.6)$ & 0.12 & 0.35 & $6.0(1.4)$ & $7.5( \pm 2.1)$ & $0.00^{\dagger}$ & 0.85 \\
\hline \multicolumn{9}{|c|}{$\begin{array}{l}\mathrm{TA}=\text { transversus abdominis; } \mathrm{OI}=\text { oblique abdominis internus; } \mathrm{OE}=\text { oblique abdominis externus; } \mathrm{ADIM}=\text { abdominal drawing-in manoeuvre; } \mathrm{ASLR}-\mathrm{L}=\text { active straight-leg raise (left); } \\
\mathrm{ASLR}-\mathrm{R}=\text { active straight-leg raise (right); } \mathrm{SD}=\text { standard deviation. }\end{array}$} \\
\hline
\end{tabular}

line through the middle of the image. Measurements were conducted perpendicular to the muscle fascia. ${ }^{[10,11]}$

\section{Data analysis}

Statistical analysis was conducted using Statistica (version 10). Thickness was reported as absolute muscle thickness at rest (TA, OI and $\mathrm{OE}$ ) and thickness percentage change or muscle activity (TA and OI). Thickness percentage change was calculated as muscle thickness during activity as a ratio to muscle thickness at rest: muscle thickness in contracted state minus muscle thickness at rest, divided by muscle thickness at rest multiplied by $100 .^{[10,11]}$ Absolute thickness as well as thickness change on the dominant and non-dominant sides were compared using the paired Student's $t$-test. Likewise, the absolute thickness and thickness change recorded at the start of the cricket season were compared to thickness measurements at the end of the cricket season using the paired Student's $t$-test. ${ }^{[7]}$ Statistical significance was set at $p<0.05$. Effect sizes were calculated using Cohen's $d$ where effect sizes of 0.2, 0.5 and 0.8 were interpreted as small, medium and large, respectively.

\section{Results}

Twenty-six healthy, male, right-handed, fast, fast-medium and medium pace bowlers aged 18 - 26 years participated in the study (mean age 21.8 years, standard deviation $(\mathrm{SD}) \pm 1.8$ ). Most had more than 6 years' experience as a pace bowler, with the exception of two who each had 5 years' experience.

The absolute thickness of the non-dominant OI at rest was higher than that of the dominant OI (Table 1), with large effect sizes found for pre- $(\mathrm{ES}=0.87)$ and post-season ( $\mathrm{ES}=1.09)$ measurements $(p=0.001)$. No side-to-side difference was found in absolute muscle thickness at rest for TA ( $p=0.25$ and $p=0.10)$ and $\mathrm{OE}(p=0.33$ and $p=0.76)$ at the start or end of the season, respectively. At the start of the season the percentage change during the ADIM, thus muscle activity, was higher for the non-dominant OI than for the dominant $\mathrm{OI}(p=0.02$; $\mathrm{ES}=0.51)$. This was not the case when looking at post-season OI activation during $\operatorname{ADIM}(p=0.37)$.

Absolute thickness of the dominant OE at rest was significantly higher at the end of the season compared with at the start of the season ( $p=0.001 ; \mathrm{ES}=0.85)$, while no difference was found in TA $(p=0.07)$ and OI ( $p=0.28$ ) thickness (Table 2$)$. Furthermore, no difference was found in recruitment of the dominant and non-dominant TA and OI in ADIM ( $p=0.07-0.75)$, ASLR-L $(p=0.07-0.88)$ and ASLR-R $(p=0.06$ - 0.67) activity positions at the start compared with the end of the cricket season.

During ASLR-R, the activity of the left TA was significantly higher than that of the right TA during ASLR-L $(p=0.03)$ at the end of the season (Fig. 1c). The same was true for TA during the ASLR-L ( $p=0.17)$ at the start of the season (Fig. 1a). During ipsilateral muscle activity in ASLR-L and ASLR-R, the activation of the left TA was higher than that of the right TA (pre-season: $p=0.79$; post-season: $p=0.15$ ).

\section{Discussion}

We investigated the absolute thickness of TA, OI and OE at rest as well as TA and OI activity in ADIM, ASLR-L and ASLR-R activity at the start and end of a cricket season. In our study, OI was the thickest muscle and TA the thinnest, which is similar to the findings of Mannion et al. ${ }^{[5]}$ and Rankin et al. ${ }^{[7]}$ The absolute muscle thickness of TA $(4.6-5.3 \mathrm{~cm})$ was slightly greater than that found by Mannion et al..$^{[5]}(3.9-4.0 \mathrm{~cm})$, while the OI values $(11.3-15.2 \mathrm{~cm})$ in the present study were much higher 


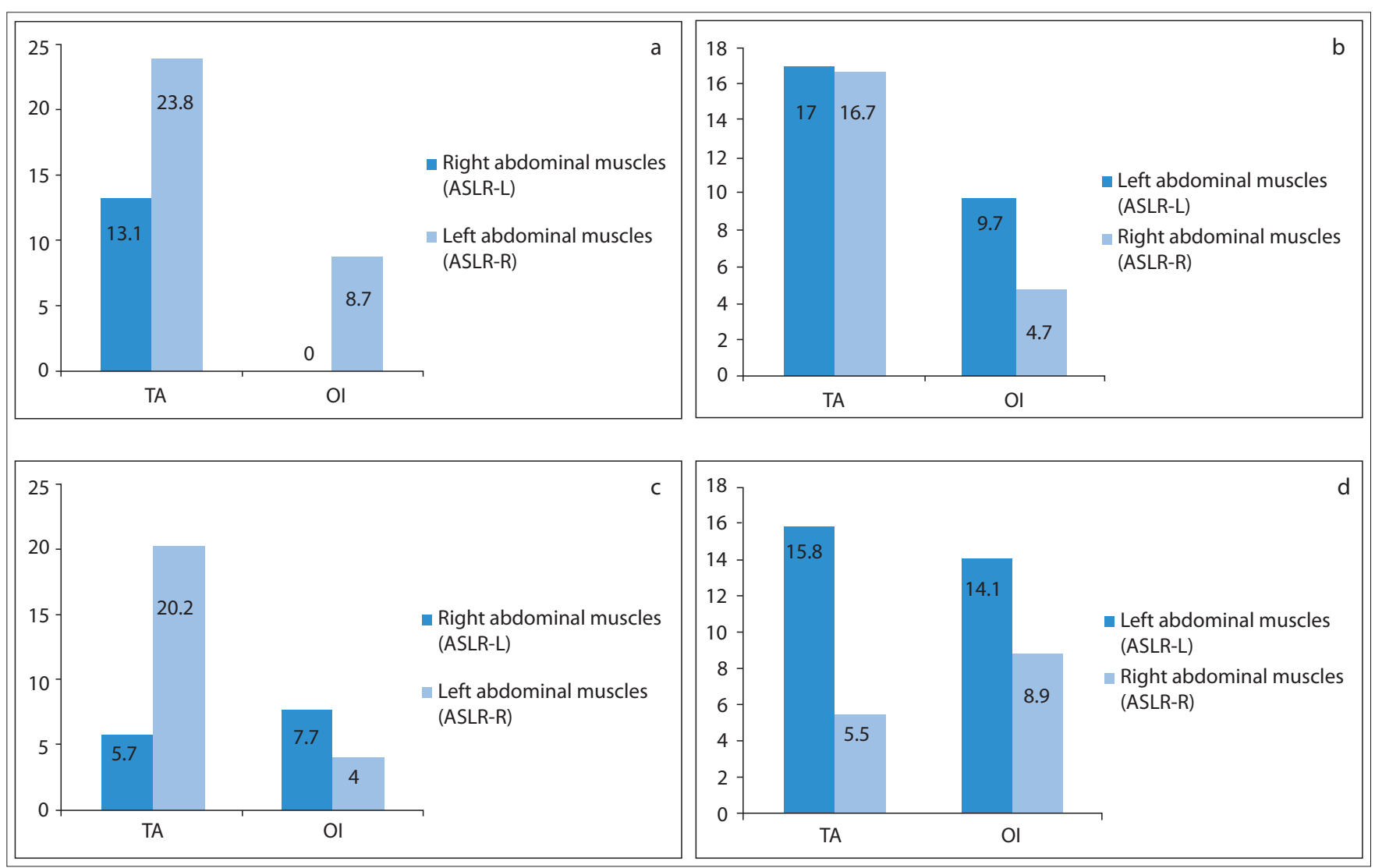

Fig. 1. Percentage change in thickness measured in the ASLR-L (non-dominant) and ASLR-R (dominant) activity positions: (a) pre-season in contra-lateral muscles (TA: $\mathrm{p}=0.17$; OI: $\mathrm{p}=0.13$ ); (b) pre-season in ipsilateral muscles (TA: $\mathrm{p}=0.79 ;$ OI: $\mathrm{p}=0.48)$; (c) post-season in contra-lateral muscles (TA: $\mathrm{p}=0.03$; OI: $\mathrm{p}=0.50) ;(d)$ post-season in ipsilateral muscles (TA: $\mathrm{p}=0.15 ;$ OI: $\mathrm{p}=0.31$ ).

and the $\mathrm{OE}$ values $(6.0-7.5 \mathrm{~cm})$ correspond closely to those found by Mannion et al. (OI: $8.3-8.6 \mathrm{~cm}$; OE: $6.7-7.1 \mathrm{~cm}$ ). Mannion et al. ${ }^{[5]}$ used a non-sporting population consisting of volunteers and this may explain their participants' lesser TA and OI thickness values. In contrast, Rankin et al. ${ }^{[7]}$ found a mean TA thickness $(4.5-5.1 \mathrm{~cm})$ similar to what was found in the present study, with thinner OI $(11.7-11.8 \mathrm{~cm})$ and thicker OE muscles $(9.6-9.7 \mathrm{~cm})$ in moderately active volunteers. ${ }^{[7]}$ Although Hides et al..$^{[8]}$ did not measure OE thickness, they found higher thickness values for both TA $(6.8-7.2 \mathrm{~cm})$ and OI $(16.7-16.8$ $\mathrm{cm})$ than in this study. The professional fast bowlers assessed in the latter study were likely to be able to devote more time to pace-bowling training and conditioning, which may lead to greater hypertrophy of the abdominal musculature than in elite players. These differences in OI absolute thickness suggest that the more active the population, the larger the OI values, and might indicate the particular activation of $\mathrm{OI}$ muscles during high load, repeated, asymmetrical physical activity. A similar trend was found in resting thickness for TA, but not for OE. It would appear that as the OI gets thicker, the OE gets thinner, thus the relative balance in resting muscle thickness changes as the activity of the individual changes. This hypothesis requires further study; however, the consistent methods used by the latter studies ${ }^{[5,7,8]}$ might suggest that different abdominal muscles display far more complex responses to training than was previously thought.

In the current study, the absolute thickness of the OI at rest was significantly higher on the non-dominant side than on the dominant side, both at the start as well as at the end of the cricket season as shown in Table 1. The large effect sizes emphasise the significance of this finding. In contrast to the findings in our study, Mannion et $a l .{ }^{[5]}$ and Springer et al ${ }^{[6]}$ found no statistically significant differences between the left and right abdominal muscle thickness and Rankin $e t$ al. ${ }^{[7]}$ found asymmetries in TA thickness only. In the case of Springer et al. ${ }^{[6]}$ and Mannion et al. ${ }^{[5]}$ the researchers used a sample where no or few participants engaged in unilateral or rotational sporting activities. Springer et al. ${ }^{[6]}$ suggest that individuals who routinely participate in rotational activities such as tennis and golf may be more likely to display asymmetries. Asymmetrical findings may increase the risk of developing pathology. ${ }^{[1]}$ However, in a mathematical model used to estimate lumbar spinal stresses during quadratus lumborum muscle asymmetry, it was suggested that quadratus lumborum muscle asymmetry only causes small stresses and it may even help to reduce stress on the lumbar spine. ${ }^{[12]}$ Participants in this study were all healthy, pain and injury-free at the time of testing, which may suggest that factors other than pathology are responsible for the asymmetries found, as suggested by De Visser et al. ${ }^{[12]}$ These differences in absolute muscle thickness on the non-dominant and dominant sides may be as a result of long-term preferential use of the right bowling arm and subsequent preferential, asymmetrical recruitment of abdominal muscle fibres that may play a role in protection of the lumbar spine. It should, however, be noted that the asymmetries seen in pace bowlers are one of the factors predisposing them to their known susceptibility to lower-back injury, ${ }^{[1]}$ 


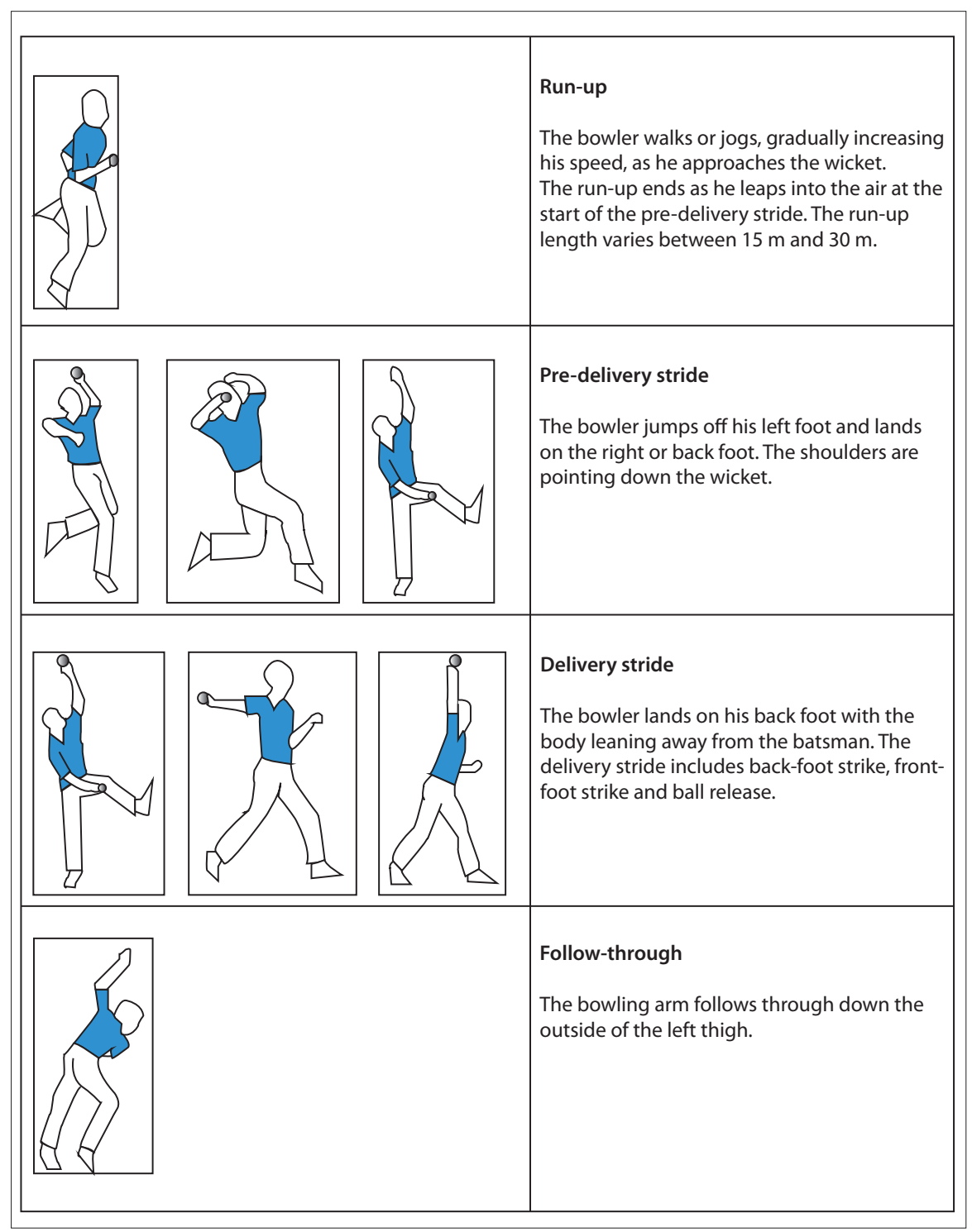

Fig. 2. The phases of the pace bowling action (reference made to a right-handed bowler).

but this asymmetry may also play a protective role. $^{[12]}$

Taking the biomechanics of the pace-bowling action into account (Fig. 2), weight is put onto the non-dominant leg at front-foot placement when the ground reaction force is extremely high, ${ }^{[13]}$ which may mean that the ipsilateral OI has to contract to assist in absorbing the ground reaction forces, taking into account the position of the pace bowler. In the present study, the absolute thickness of the non-dominant OI was greater than that of the dominant OI, which fits with the above theory. Also, while the non-dominant leg takes a vast amount of the load during the delivery stride, the dominant $\mathrm{OE}$ contracts in order to stabilise the pelvis in its typical cross-over activation fashion. ${ }^{[14]}$ The repetitive nature of and TA during the ADIM show that the abdominal muscles on the non-dominant side are activated at a higher rate than the dominant abdominal muscles. This may emphasise the repeated utilisation of the non-dominant TA and OI during the bowling action, and the subsequent preferential recruitment. The asymmetry of the bowling action may play a role in the development of habitual movement patterns maintained by pre-programmed motor-control pathways. ${ }^{[15]}$ Furthermore, the activation of TA is higher than the activation of OI in the ADIM, ASLR-L and ASLR-R activity positions, which indicates that the TA is the preferential muscle recruited during sub-maximal muscle contraction. ${ }^{[10]}$

During asymmetrical activity positions, the left TA showed statistically significantly greater activity during the ASLR-R than the right TA during ASLR-L (Fig. 1). This was also clear during the ASLR-L, where the left TA activity was again much higher than the right TA activity during ASLR-R; although not statistically significant, it is of clinical significance. This finding indicates that the left TA activity is higher than the right TA activity during contra-lateral as well as ipsilateral activity positions. This is contradictory to a study by Teyhen et al. ${ }^{[11]}$ which found that the TA is symmetrically activated during asymmetrical activity, while $\mathrm{Hu}$ et al. ${ }^{[14]}$ found that TA and OI showed greater asymmetrical activity than $\mathrm{OE}$. The difference in results can be attributed to the different populations studied, where Teyhen et al. ${ }^{[11]}$ studied Department of Defence healthcare beneficiaries, including active-duty military family members and retirees, and $\mathrm{Hu}$ et al. ${ }^{[14]}$ studied a group of females. Neither author specified details on specific sports participation. The difference in dominant and non-dominant TA activity is less pronounced during the pre-season measurements, which again shows that the intense training and repetition of the asymmetrical bowling action that takes place during a cricket season may be responsible for the preferential increase in activation of non-dominant TA.

\section{Study limitations}

No comparison group of non-athletes was assessed in parallel to the pace bowlers in this study. Generalisation of the current findings is thus limited to cricket pace bowlers and future studies should be performed to 
compare abdominal muscle absolute thickness and activity in different sporting populations. Furthermore, studies should be done to assess the training components of the cricket season, the activation of the abdominal muscles during the pace-bowling action and its influence on abdominal muscle thickness.

\section{Conclusion}

Our study highlights the possible muscle adaptations in absolute muscle thickness and activity as a consequence of the high load of asymmetrical bowling action that is performed repeatedly during matches and training. The type and intensity of training that took place during the cricket season may have accounted for the asymmetries in abdominal muscle thickness that were found at the end of the season.

Acknowledgement. Funding was granted by the Carnegie Foundation, the South African National Research Foundation and the South African Society of Physiotherapy.

\section{References*}

1. Engstrom CM, Walker DG, Kippers V, Mehnert AJ. Quadratus lumborum asymmetry and L4 pars injury in fast bowlers: A prospective MR study. Med Sci Sports Exerc 2007;39:910-917. [http://dx.doi.org/10.1249/mss.0b013e3180408e25]

2. Panjabi MM. The stabilizing system of the spine. Part II. Neutral zone and instability hypothesis. J Spinal Disord 1992;5:390-396. [http://dx.doi.org/10.1097/00002517199212000-00002]

3. Ferreira PH, Ferreira ML, Nascimento DP, Pinto RZ, Franco MR, Hodges PW. Discriminative and reliability analyses of ultrasound measurement of abdominal muscles recruitment. Man Ther 2011;16:463-469. [http://dx.doi.org/10.1016/j. math.2011.02.010]

4. Hides J, Wilson S, Stanton W, et al. An MRI investigation into the function of the transversus abdominis muscle during "drawing-in" of the abdominal wall. Spine (Phila Pa 1976) 2006;31:E175-E178. [http://dx.doi.org/10.1097/01.brs.0000202740.86338.df]
5. Mannion AF, Pulkovski N, Toma V, Sprott H. Abdominal muscle size and symmetry at rest and during abdominal hollowing exercises in healthy control subjects. J Anat 2008;213:173-182. [http://dx.doi.org/10.1111/j.1469-7580.2008.00946.x]

6. Springer BA, Mielcarek BJ, Nesfield TK, Teyhen DS. Relationships among lateral abdominal muscles, gender, body mass index, and hand dominance. JOSPT 2006;36:289-297.

7. Rankin G, Stokes M, Newham DJ. Abdominal muscle size and symmetry in normal subjects. Muscle Nerve 2006;34:320-326. [http://dx.doi.org/10.1002/mus.20589]

8. Hides J, Stanton W, Freke, M, Wilson, S, McMahon, S, Richardson, C. MRI study of the size, symmetry and function of the trunk muscles among elite cricketers with and without low back pain. Br J Sports Med 2008;42:809-813. [http://dx.doi.org/10.1136/ bjsm.2007.044024]

9. Teyhen DS, Gill NW, Whittaker JL, Henry SM, Hides JA, Hodges P. Rehabilitative ultrasound imaging of the abdominal muscles. JOSPT 2007;37:450-466.

10. Teyhen DS, Bluemle LND, Baker SE, et al. Changes in lateral abdominal muscle thickness during the abdominal drawing-in maneuver in those with lumbopelvic pain. JOSPT 2009;39:791-798. [http://dx.doi.org/10.2519/jospt.2009.3128]

11. Teyhen DS, Williamson JN, Carlson NH, et al. Ultrasound characteristics of the deep abdominal muscles during the active straight leg raise test. Arch Phys Med Rehabi 2009;90:761-767. [http://dx.doi.org/10.1016/j.apmr.2008.11.011]

12. De Visser H, Adam CJ, Crozier S, Pearcy MJ. The role of quadratus lumborum asymmetry in the occurrence of lesions in the lumbar vertebrae of cricket fast bowlers. Med Eng Phys 2007;29:877-885. [http://dx.doi.org/10.1016/j. medengphy.2006.09.010

13. Ferdinands RE, Kersting U, Marshall RN. Three-dimensional lumbar segment kinetics of fast bowling in cricket. J Biomech 2009;42:1616-1621. [http://dx.doi.org/10.1016/j. jbiomech.2009.04.035]

14. $\mathrm{Hu} \mathrm{H}$, Meijer OG, Hodges PW, et al. Understanding the active straight leg raise (ASLR): An electromyographic study in healthy subjects. Man Ther 2012;17:531-537. [http://dx.doi.org/10.1016/j.math.2012.05.010]

15. Davey NJ, Lisle RM, Loxton-Edwards B, Nowicky AV, McGregor AH. Activation of back muscles during voluntary abduction of the contralateral arm in humans. Spine (Phila Pa 1976) 2002;27:1355-1360. [http://dx.doi.org/10.1097/00007632-20020615000019]

* A more comprehensive reference list may be obtained from the corresponding author. 